\title{
ON INFINITE SERIES OF SETS
}

\author{
by DAVID ELLIS
}

(Received 30th March, 1954)

1. Introduction. We continue our studies $(2,3,4,5)$ of the algebraic, geometric, and analytical similarities and contrasts between Boolean algebras and the real field. In this note we contrast the convergence of series in set algebras with that in the real field.

One considers the Boolean algebra $\mathcal{B}$ of all subsets of a given set $S$. By addition, one means symmetric difference $A+B=(A \cap(S-B)) \cup((S-A) \cap B)$. As usual, one writes

$$
\sum_{i=1}^{n} A_{i} \text { for } A_{1}+A_{2}+\ldots+A_{n} \text {. }
$$

One introduces a topology into $\mathfrak{B}$ (the Kantorovitch (6) or sequential order topology (1)) by defining $\varlimsup_{i \rightarrow \infty} A_{i}=\bigcap_{i=1}^{\infty} \bigcup_{j=i}^{\infty} A_{j}, \varliminf_{i \rightarrow \infty} A_{i}=\bigcup_{i=1}^{\infty} \bigcap_{j=i}^{\infty} A_{j}$ and then $\lim _{i \rightarrow \infty} A_{i}=A$ if and only if

$$
\varliminf_{i \rightarrow \infty} A_{i}=\varlimsup_{i \rightarrow \infty} A_{i}=A \text {. }
$$

The sum of an infinite series $\sum_{i=1}^{\infty} A_{i}$ is then defined to be $\lim _{n \rightarrow \infty} \sum_{i=1}^{n} A_{i}$, provided this limit exists. The series is called convergent if it has a sum and in this case the sum and the series itself are denoted by the same symbol $\sum_{i=1}^{\infty} A_{i}$. The interesting contrast shown in the present note is as follows: In the real field, a necessary but far from sufficient condition that a series converge is that the sequence of its terms have limit zero. In $\mathfrak{B}$, however, this condition is necessary and sufficient.

2. The main result.

LEMMA 1. $\sum_{i=1}^{n} A_{i}$ consists of those points (elements of $S$ ) which are in exactly an odd number of the sets $A_{i} ; i=1,2, \ldots, n$.

Proof. This result is well-known (7) and is easily proved by mathematical induction.

Lemma 2. $\varlimsup_{i \rightarrow \infty} A_{i}$ and $\varliminf_{i \rightarrow \infty} A_{i}$ consist, respectively, of those points which are in infinitely many and those points which are in almost all (all but a finite number), respectively, of the sets $A_{i} ; i=1,2, \ldots$.

Proof. These results are well-known (1) and are quite clear from the definitions of the superior and inferior limits.

LEMMA 3. If $\sum_{i=1}^{\infty} A_{i}$ is convergent, then no point is in infinitely many of the sets $A_{i} ; i=1,2, \ldots$.

Proof. Suppose $p \epsilon A_{i}$ for infinitely many $i$. Then $p \in \sum_{i=1}^{n} A_{i}$ for infinitely many $n$ (one merely takes for $n$ every alternate value of $i$ for which $p \in A_{i}$ and applies Lemma 1). However, $p$ is not in almost all $\sum_{i=1}^{n} A_{i}$ since $p \in \sum_{i=1}^{m} A_{i}$ implies $p \notin \sum_{i=1}^{n} A_{i}$, where $n$ is the first index exceeding $m$ 
for which $p \in A_{n}$. Thus, $p \in \varlimsup_{n=\infty} \sum_{i=1}^{n} A_{i}$ but $p \notin \varliminf_{n \rightarrow \infty} \sum_{i=1}^{n} A_{i}$ so that the series cannot converge. This proves the contrapositive of Lemma 3. null set).

Lemma 4. A necessary condition that $\sum_{i=1}^{\infty} A_{i}$ converge is that $\lim _{i \rightarrow \infty} A_{i}=0$ (0 denotes here the

Proof. If the series is convergent, $\varlimsup_{i \rightarrow \infty} A_{i}=0$, by Lemmas 2 and 3. However,

so that $\lim _{i \rightarrow \infty} A_{i}=0$.

$$
0 \subset \varlimsup_{i \rightarrow \infty} A_{i} \subset \varlimsup_{i \rightarrow \infty} A_{i}=0
$$

LEMma 5. If $\lim _{i \rightarrow \infty} A_{i}=0$, then $\sum_{i=1}^{\infty} A_{i}$ converges.

Proof. Suppose $\lim _{i \rightarrow \infty} A_{i}=0$. Then no point is in infinitely many of the $A_{i} ; i=1,2, \ldots$. Thus, if $p \in \varlimsup_{n \rightarrow \infty} \sum_{i=1}^{n} A_{i}$, there is a last $A_{i}$, say $A_{k}$, with $p \in A_{i}$. Thus $p \in A_{i}$ for exactly an odd number of $i \leqslant k$ and $p \nmid A_{i}$ for $i>k$. Then $p \in \sum_{i=1}^{n} A_{i}$ for all $n>k$. Thus,

and the series converges.

$$
\varliminf_{n \rightarrow \infty} \sum_{i=1}^{n} A_{i}=\varlimsup_{n \rightarrow \infty} \sum_{i=1}^{n} A_{i}=\varliminf_{n \rightarrow \infty} \sum_{i=1}^{n} A_{i}
$$

Theorem 1. The series $\sum_{i=1}^{\infty} A_{i}$ converges if and only if $\lim _{i \rightarrow \infty} A_{i}=0$ and in this case the sum of the series is that subset of $S$ each of whose points lies in exactly an odd number of the $A_{i} ; i=1,2, \ldots$.

Proof. Immediate from Lemmas 1, 4, and 5.

3. Representing series. We employ the Boolean ring notation (1) and write $A B$ for $A \cap B$ here desired. A sequence $A_{1}, A_{2}, \ldots$ in $\mathcal{B}$ is called a representing series if $\sum_{i=1}^{\infty} X_{i} A_{i}$ converges for every choice of the coefficients $X_{i}$ and, furthermore, every subset of $S$ (that is, every member of $\mathfrak{B}$ ) is the sum of this series for some choice of the coefficients $X_{i}$. The sequence $A_{1}, A_{2}, \ldots$ is called a partition sequence of $S$ if $\bigcup_{i=1}^{\infty} A_{i}=S$ and $\bigcap_{j=1}^{\infty} A_{i_{j}}=0$ for every infinite subsequence $\left\{A_{i_{j}}\right\}$ of $\left\{A_{i}\right\}$. Countable partitions of $S$ are, obviously, special cases of partition sequences.

Theorem 2 . A sequence $A_{1}, A_{2}, \ldots$ is a representing series in $\mathcal{B}$ if and only if it is a partition sequence of $S$.

Proof. Suppose $\left\{A_{i}\right\}$ is a representing series. Taking $X_{i}=S$, for all $i, \sum_{i=1}^{\infty} A_{i}$ converges and, by Theorem 1, $\lim _{i \rightarrow \infty} A_{i}=0$. Hence, $\bigcap_{j=1}^{\infty} A_{i_{j}}=0$ for every subsequence $\left\{A_{i j}\right\}$, by Lemma 2. Also, $\sum_{i=1}^{\infty} X_{i} A_{i}=S$ for some. choice of the $X_{i}$. Then $\sum_{i=1}^{\infty} X_{i} A_{i} \subset \bigcup_{i=1}^{\infty} X_{i} A_{i} \subset \bigcup_{i=1}^{\infty} A_{i}$. Thus, $\left\{A_{i}\right\}$ is a partition sequence of $S$. Suppose, on the other hand, that $\left\{A_{i}\right\}$ is a partition sequence. Then 
$\bigcap_{j=1}^{\infty} A_{i_{j}}=0$ for any subsequence $\left\{A_{i_{j}}\right\}$ so that $\varlimsup_{i \rightarrow \infty} A_{i}=0$, by Lemma 2 , and, hence, $\lim _{i \rightarrow \infty} X_{i} A_{i}=0$ for any choice of the $X_{i}$. By Theorem $1, \sum_{i=1}^{\infty} X_{i} A_{i}$ converges. Since $\bigcup_{i=1}^{\infty} A_{i}=S, \bigcup_{i=1}^{\infty} B_{i}=S$, where $B_{i}$ is $A_{i} \cap\left(S-\bigcup_{j=1}^{i-1} A_{j}\right)$. The $B_{i}$ are, however, disjoint so that $\sum_{i=1}^{\infty} B_{i}=\bigcup_{i=1}^{\infty} B_{i}$. Thus, $\sum_{i=1}^{\infty} B_{i}=S$. Let $T=S$. Then $T=\sum_{i=1}^{\infty}\left(T\left(S-\bigcup_{j=1}^{-1} A_{j}\right)\right) A_{i}$ and $\left\{A_{i}\right\}$ is a representing series.

There are, of course, no representing series in the real field and, although

$$
\bigcup_{i=1}^{\infty} a_{i}=\lim _{n \rightarrow \infty} \sum_{k=1}^{n} s_{k, n}\left(a_{i}\right)
$$

is definable in analogy to the Boolean case (where $s_{k, n}\left(a_{i}\right)$ is the $k$ th elementary symmetric function of $\left.a_{1}, a_{2}, \ldots, a_{n}\right)$, there is no analogue to the condition $\bigcup_{i=1}^{\infty} a_{i}=S$ and, hence, no partition sequence of reals.

4. Series of Boolean functions. One recalls (1) that any Boolean function of one variable $X$ (that is, a function finitely defined in terms of the Boolean operations on $X$ and constant elements of 3 ) may be written in the conjunctive normal form :

or, in ring notation,

$$
F(X)=(A \cap X) \cup(B \cap(S-X))
$$

$$
F(X)=(A+B) X+B
$$

where $A$ and $B$ are constants. One says, as usual, that a series of functions $\sum_{i=1}^{\infty} F_{i}(X)$ converges if the series of functional values converges for all values of $X$. In the real field, a series of polynomials (the analogue in the reals of Boolean functions) may converge to a function which is not a polynomial. We observe, however

Theorem 3. A series $\stackrel{\infty}{\Sigma} F_{i}(X)$ of Boolean functions in $\mathcal{B}$ converges if and only if $\lim _{i \rightarrow \infty} A_{i}=\lim _{i \rightarrow \infty} B_{i}=0$, where $F_{i}(X)=\left(A_{i}+B_{i}\right) X+B_{i}$, and, in this case, the sum function is the Boolean function

$$
F(X)=\left(\sum_{i=1}^{\infty} A_{i}+\sum_{i=1}^{\infty} B_{i}\right) X+\sum_{i=1}^{\infty} B_{i}
$$

Proof. If $\sum_{i=1}^{\infty} F_{i}(X)$ converges for all $X, \sum_{i=1}^{\infty} F_{i}(0)=\sum_{i=1}^{\infty} B_{i}$ converges and $\lim _{i \rightarrow \infty} B_{i}=0$, by Theorem 1. Also, $\sum_{i=1}^{\infty} F_{i}(S)=\sum_{i=1}^{\infty} A_{i}$ converges and $\lim _{i \rightarrow \infty} A_{i}=0$, again by Theorem 1 . Using the sequential continuity (1) of Boolean operations in the Kantorovitch topology, we have

$$
\text { (†) } \lim _{n \rightarrow \infty}\left(\left(\sum_{i=1}^{n} A_{i}+\sum_{i=1}^{n} B_{i}\right) X+\sum_{i=1}^{n} B_{i}\right)=\left(\lim _{n \rightarrow \infty} \sum_{i=1}^{n} A_{i}+\lim _{n \rightarrow \infty} \sum_{i=1}^{n} B_{i}\right) X+\lim _{n \rightarrow \infty} \sum_{i=1}^{n} B_{i}
$$

provided the limits on the right hand side exist. If, on the other hand, $\lim _{i \rightarrow \infty} A_{i}=\lim _{i \rightarrow \infty} B_{i}=0$, then the limits on the right hand side of $(\dagger)$ exist, according to Theorem 1 , and $\sum_{i=1}^{\infty} F_{i}(X)$ converges to the prescribed sum function. 
5. Additional comments.

(1) Using the representation theorems (1) of Loomis and others, one may attack infinite series in general $\sigma$-complete Boolean algebras from the results given in this note.

(2) One may generalize the results of this note in set algebras by considering the full order topology (1) and considering Moore-Smith series (nets of partial sums) rather than sequential series.

(3) One may generalize our notion of partition sequence as follows : A pseudo-partition of a set $S$ is a collection of subsets of $S$ whose join (set-theoretic sum) is $S$ and having the property that each point of $S$ lies in only finitely many of the members of the pseudo-partition. A theory of the relations arising from pseudo-partitions might be an interesting generalization of the theory of equivalence relations arising from ordinary partitions (1).

(4) One might also find interesting a study of infinite joins of numbers

$$
\bigvee_{i=1}^{\infty} a_{i}=\lim _{n \rightarrow \infty} \sum_{k=1}^{n} s_{k, n}\left(a_{i}\right)
$$

defined in analogy with the join operation in Boolean rings

(in a Boolean ring, $A \vee B=A+B+A B, A \vee B \vee C=A+B+C+A B+A C+B C+\dot{A} B C$, etc.).

\section{REFERENCES}

(1) Birkhoff, Garrett, Lattice Theory (Revised Edition), American Mathematical Society, New York, 1948.

(2) Ellis, David, "Autometrized Boolean Algebras," I and II, Canadian Jour. of Math., vol. 3 (1951), pp. 87-93 and 145-147. 92-95.

(3) Ellis, David, Notes on Abstract Distance Geometry, III, Simon Stevin, vol. 29 (1952), pp.

(4) Ellis, David, "Remarks on Boolean functions." To appear in Jour. of the Math. Soc. of Japan.

(5) Ellis, David and Sprinkle, H. D., Topology in B-metric spaces. In preparation.

(6) Kantorovitch, L. V., " Lineare halbgeordnete Räume," Mat. Sbornik, vol. 44 (1937), pp. 121-165.

(7) Vaidyanathaswamy, R., Treatise on Set Topology, I, Indian Math. Soc., Madras, 1947.

\section{Department of Mathematics, \\ UNIVERSITY OF FLORIDA}

